Pacific Journal of Mathematics

REDUCIBLE SUMS OF SIMPLE MULTIVECTO 


\section{IRREDUCIBLE SUMS OF SIMPLE MULTIVECTORS}

\section{Herbert Busemann and D. Edward Glassco II}

Denoting by $V^{n}(F)$ the $n$-dimensional vector space over the field $F$ of characteristic 0 , let $V_{r}^{n}(F)$ be the linear space of all $r$-vectors $\tilde{R}$ over $V^{n}(F)$ and $G_{r}^{n}(F)$ the Grassmann cone of the simple $r$-vectors $R$ in $V_{r}^{n}(F)$. The sum $\tilde{R}=\sum_{i=1}^{k} R_{i}\left(R_{i} \in\right.$ $\left.G_{r}^{n}(F)\right)$ is irreducible if $\tilde{R}$ is not the sum of fewer than $k$ elements of $G_{r}^{n}(F)$. (Duality reduces the interesting cases to $2 \leqq r \leqq n / 2$.) Such sums are trivial only for $r=2$, because $\bigwedge_{i=1}^{k} R_{i} \neq 0$ while always sufficient for irreducibility is then also necessary. Extension of $F$ does not influence irreducibility if $r=2$ but it can for $r>2$.

The sets $W_{r}^{n}(F, k)$ of those $\tilde{R}$ in $V_{r}^{n}(F)$ which are irreducible sums of $k$ terms behave as expected when $r=2$, but have the most surprising properties for larger $r$. Although $V_{3}^{6}(F)=\bigcup_{k=1}^{3} W_{3}^{6}(F, k)$ and $W_{3}^{6}(F, 3) \neq \phi$, the sets $W_{3}^{\ell}(\boldsymbol{R}$ or $C, 2)$ have interior points as sets in $V_{3}^{6}(R$ resp. $C)$ and so does $W_{3}^{\bullet}(\boldsymbol{R}, 3)$ but $W_{3}^{6}(\boldsymbol{C}, 3)$ does not.

The paper is based on the thesis [1] with the same title by the second author.

The smallest number $k$ for which $V_{r}^{n}(F, k)=\bigcup_{i=1}^{k} W_{r}^{n}(F, i)$ coincides with $V_{r}^{n}(F)$ is denoted by $N(F, n, r)$ which by duality equals $N(F, n$, $n-r)$. Obviously $N(F, n, r) \leqq\left(\begin{array}{l}n \\ r\end{array}\right)$. But in spite of various inequalities relating these numbers which show that $\left(\begin{array}{l}n \\ r\end{array}\right)$ is much too large, the precise value of $N(F, n, r)$ is known only in the two cases implied by the above statements: namely $N(F, n, 2)=[n / 2]$ and $N(F, 6,3)=3$.

The values $N(\boldsymbol{C}, 7,3)=5, N(\boldsymbol{C}, 8,3)=7$, and $N(\boldsymbol{C}, 9,3)=10$ have been claimed but questioned, see Schouten [3, p. 27] and [1].

The purpose of our investigation is to elucidate why the case $r=2$ is so much simpler than $2<r<n-2$. In addition to the already mentioned facts we show that $V_{2}^{n}(F, k)$ is an algebraic variety, because, if $\widetilde{R}^{(i)}$ is the $i$ th exterior power of $\widetilde{R}$, then $\widetilde{R}^{(k+1)}=0$ is necessary and sufficient for $\widetilde{R} \in V_{r}^{n}(F, k)$ when $r=2$, but merely necessary when $r>2$. This implies $\operatorname{dim} V_{2}^{n}(\boldsymbol{R} \operatorname{resp.} \boldsymbol{C}, k)<\operatorname{dim} V_{2}^{n}(\boldsymbol{R} \operatorname{resp}$. $C, k+1)$ for $1 \leqq k<[n / 2]$ in contrast to the case $n=6, r=3$. In fact we show that $V_{r}^{n}(\boldsymbol{R}$ or $C, k)$ is for $r>2, k>1$, and $n \geqq(k-1) r+3$ not even a closed set.

An irreducible representation $\widetilde{R}=\sum_{i=1}^{k} R_{i}, k>1$, is for $r=2$ never unique, but for $r>2$ it is (up to a permutation) if $\bigwedge_{i=1}^{k} R_{i} \neq 0$ and $k \leqq r$. The condition $k \leqq r$ is probably superfluous but enters -like $n \geqq(k-1) r+3$ (instead of $n \geqq r+3$ ) above-because we use the Plücker relations for simple vectors which get out of hand for 
large $k$. A coordinate-free approach would therefore be preferable, but in many cases we were not able to devise one.

We will continue using capitals $(R, S, T)$ with a tilde and with or without subscripts for general multivectors and omit the tilde only when the vectors are known or assumed to be simple.

2. Results for general $F, n, r, k$. The following agreement will prove convenient. $e_{1}, e_{2}, \ldots$ are used for elements of a base. If two spaces $V^{m} \subset V^{n}$ occur, then the base $e_{1}, \cdots, e_{n}$ of $V^{n}$ is chosen so that $e_{1}, \cdots, e_{m}$ is a base of $V^{m}$. We begin with some simple remarks.

(2.1) If $R \in G_{r}^{n}$ then $R=R^{\prime}+S \wedge e_{n}$ with $R^{\prime} \in G_{r}^{n-1}$ and $S \in$ $G_{r-1}^{n-1}$.

For, with suitable $v_{i} \in V^{n-1}$ and $\beta_{i}$

$$
\begin{aligned}
R & =\bigwedge_{i=1}^{r}\left(v_{i}+\beta_{i} e_{n}\right) \\
& =\bigwedge_{i=1}^{r} v_{i}+\left[\sum_{i=1}^{r}(-1)^{n-i} \beta_{i} v_{1} \wedge \cdots \wedge v_{i-1} \wedge v_{i+1} \wedge \cdots \wedge v_{r}\right] \wedge e_{n} .
\end{aligned}
$$

If the $v_{i}$ are dependent, the bracket reduces to one term; if not, the bracket is an $(r-1)$-vector in the $r$-space spanned by $v_{1}, \cdots, v_{r}$ and hence is simple.

We apply (2.1) to prove

(2.2) $\widetilde{R} \in W_{r}^{n}(F, k)$ if and only if $\widetilde{R} \wedge e_{n+1} \wedge \cdots \wedge e_{n+m} \in W_{r+m}^{n+m}(F, k)$.

It suffices to prove this for $m=1$. We show if $\widetilde{R} \in W_{r}^{n}(k)$ and $\widetilde{R} \wedge$ $e_{n+1} \in W_{r+1}^{n+1}(l)$, then $l=k$. Trivially $\widetilde{R} \wedge e_{n+1} \in V_{r+1}^{n+1}(k)$, whence $l \leqq k$. By (2.1) and the hypothesis $\widetilde{R} \wedge e_{n+1}=\sum_{i=1}^{l} R_{i}=\sum_{i=1}^{l}\left(R_{i}^{\prime}+S_{i} \wedge e_{n+1}\right)$ with $R_{i} \in G_{r+1}^{n+1}, R_{i}^{\prime} \in G_{r+1}^{n}$, and $S_{i} \in G_{r}^{n}$. Therefore, $\Sigma R_{i}^{\prime}=0$ and $\widetilde{R} \wedge e_{n+1}=$ $\left(\Sigma S_{i}\right) \wedge e_{n+1}$, which implies $\widetilde{R}=\sum_{i=1}^{l} S_{i}$ and $k \leqq l$.

Corollary 2.3. $N(F, n+1, r+1) \geqq N(F, n, r)$.

Anticipating $N(F, n, 2)=[n / 2]$ we see that both equality and inequality occur. $N(2 m, 2 m-2)=N(2 m, 2)>N(2 m-1,2)=N(2 m-1$, $2 m-3)$. Similarly $N(2 m+1,2 m-1)=N(2 m, 2 m-2)$. Also $N(n, r) \geqq[(n-r+2) / 2]$, but this lower bound is for $r>2$ too small to be useful.

A consequence of (2.1) is the generalization

(2.4) If $\widetilde{R} \in V_{r}^{n}(k)$, then $\widetilde{R}=\widetilde{R}^{\prime}+\widetilde{S} \wedge e_{n}$ with $\widetilde{R}^{\prime} \in V_{r}^{n-1}(k)$ and 
$\widetilde{S} \in V_{r-1}^{n-1}(k)$.

By hypothesis $\widetilde{R}=\sum_{i=1}^{l} R_{i}\left(l \leqq k, R_{i} \in G_{r}^{n}\right)$. Applying (2.1) to each $R_{i}$ yields $\widetilde{R}=\sum_{i=1}^{l}\left(R_{i}^{\prime}+S_{i} \wedge e_{n}\right)=\sum_{i=1}^{l} R_{i}^{\prime}+\left(\sum_{i=1}^{l} S_{i}\right) \wedge e_{n}$ with $R_{i}^{\prime} \epsilon$ $G_{r}^{n-1}$ and $S_{i} \in G_{r-1}^{n-1}$, which is the assertion.

With $k=N(F, n, r)$ we deduce from (2.4):

(2.5) $N(F, n, r) \leqq N(F, n-1, r)+N(F, n-1, r-1)$.

For $r=2$ equality holds when $n$ is even and inequality holds when $n$ is odd.

A linear map $f: U^{m} \rightarrow V^{n}$ induces a homomorphism $f^{*}: U_{r}^{m} \rightarrow V_{r}^{n}$ given by $f^{*}\left(u_{1} \wedge \cdots \wedge u_{r}\right)=f\left(u_{1}\right) \wedge \cdots \wedge f\left(u_{r}\right)$. The map $f^{*}$ is surjective when $f$ is. We note

(2.6) If $f^{*}\left(R_{1}\right)+\cdots+f^{*}\left(R_{k}\right)$ is irreducible in $V_{r}^{n}$, then so is $R_{1}+\cdots+R_{k}$ in $U_{r}^{m}$.

We apply this first to the projection $f: V^{n+i} \rightarrow V^{n}$ defined by

$$
f: \sum_{i=1}^{n+k} \alpha^{i} e_{i} \longrightarrow \sum_{i=1}^{n} \alpha^{i} e_{i}
$$

and find:

(2.7) If $R_{i} \in G_{r}^{n}(F)$ and $\sum_{i=1}^{k} R_{i}$ is irreducible in $V_{r}^{n}(F)$, then it is irreducible in $V_{r}^{n+k}(F)$.

Hence

(2.8) $N(F, n+1, r) \geqq N(F, n, r)$.

The case $r=2$ shows again that both inequality and equality can occur in (2.8). Next we apply (2.6) to the map $f: V^{n+k} \rightarrow V^{n+1}$ given by

$$
f: \sum_{i=1}^{n+k} \alpha^{i} e_{i} \longrightarrow \sum_{i=1}^{n} \alpha^{i} e_{i}+\left(\sum_{i=n+1}^{n+k} \alpha^{i}\right) e_{n+1}
$$

and find using (2.2):

(2.9) If $\sum_{i=1}^{k} R_{i}$ is irreducible in $V_{r}^{n}\left(F^{\prime}\right)$, then $\sum_{i=1}^{k} R_{i} \wedge e_{n+i}$ is irreducible in $V_{r+1}^{n+k}(F)$.

Two important facts will now be proved together:

Theorem 2.10. If $\bigwedge_{i=1}^{k} R_{i} \neq 0$, then $\sum_{i=1}^{k} R_{i}$ is irreducible. The 
converse holds only for $r=2$.

THEOREM 2.11. If $\widetilde{R} \in V_{r}^{n}(F, k)$ then $\widetilde{R}^{(k+1)}=0$. The converse holds only for $r=2$.

If $r$ is odd then $\widetilde{R}^{(i)}=0$ for any $i>1$ so that $\widetilde{R}^{(k+1)}=0$ imposes no condition. If $r$ is even the relation $\left(\sum_{i=1}^{k} R_{i}\right)^{(k+1)}=0$ is obvious, so that the first part of (2.11) holds. Since

$$
\left(\sum_{i=1}^{k} R_{i}\right)^{(k)}=k ! \bigwedge_{i=1}^{k} R_{i} \text { for even } r
$$

it follows that $\sum_{i=1}^{k} R_{i} \in W_{r}^{n}(k)$ when $\bigwedge_{i=1}^{k} R_{i} \neq 0$. Applying (2.9) we see that this also holds for odd $r$.

If $\bigwedge_{i=1}^{k} R_{i}=0, r=2$, and $R_{i}=v_{i} \wedge w_{i}$ then one of the $v_{i}$ or $w_{i}$ depends on the rest, say $v_{k}=\sum_{i=1}^{k-1} \lambda_{i} v_{i}+\sum_{i=1}^{k} \mu_{i} w_{i}$ so that

$$
\sum_{i=1}^{k} R_{i}=\sum_{i=1}^{k-1}\left[v_{i} \wedge w_{i}+\left(\lambda_{i} v_{i}+\mu_{i} w_{i}\right) \wedge w_{k}\right] .
$$

Each bracket represents a simple vector because it is a 2-vector in the space spanned by $v_{i}, w_{i}$, and $w_{k}$.

That $\bigwedge_{i=1}^{k} R_{i} \neq 0$ is necessary for irreducibility only when $r=2$ follows from (2.2). This establishes (2.10).

It remains only to prove the second part of (2.11). Let $r=2$ and $\widetilde{R}^{(k+1)}=0$. Then $\widetilde{R} \in W_{r}^{n}(k+i)$ with $i \geqq 1$ is impossible because (2.10) and (2.12) would imply $\widetilde{R}^{(k+i)} \neq 0$. That $\widetilde{R}^{(k+1)}=0$ is not sufficient for $\widetilde{R} \in V_{r}^{n}(F, k)$ is obvious for odd $r$ and follows from (2.2) for even $r>2$.

Corollaries of (2.10) resp. (2.11) are:

(2.13) $N(F, n, 2)=[n / 2]$.

(2.14) If $\widetilde{R} \in W_{2}^{n}(F, k)$ then also $\widetilde{R} \in W_{2}^{n}\left(F_{0}, k\right)$ for any extension field $F_{0}$ of $F$. This is not true for $r>2$.

The latter means that for each $n-2>r>2$ there are $\widetilde{R}, k^{\prime}<$ $k, F \subset F_{0}$ with $\widetilde{R} \in W_{r}^{n}(F, k)$ and $\widetilde{R} \in W_{r}^{n}\left(F_{0}, k^{\prime}\right)$, and follows from (5.9) and (2.2). Note: The first part of (2.14) does not mean, for example, that $\widetilde{R} \in V_{2}^{n}(F), \widetilde{R} \in W_{2}^{n}\left(F_{0}, 2\right)$, hence $\widetilde{R}=R_{1}+R_{2}$ with $R_{i} \in G_{2}^{n}\left(F_{0}\right)$, imply $R_{i} \in G_{2}^{n}\left(F^{\prime}\right)$, but only that $R_{i}^{\prime} \in G_{2}^{n}(F)$ with $\widetilde{R}=R_{1}^{\prime}+R_{2}^{\prime}$ exist, compare (4.3).

Whereas in (2.2) and (2.9) the number of summands is the same in hypothesis and assertion, it is different in the next theorem which is therefore harder to prove. 
THEOREM 2.15. Let $\widetilde{R} \in W_{r}^{n}(F, k), E_{i}=\bigwedge_{l=1}^{r} e_{n+(i-1) r+l}(i=1, \cdots, j)$, then $\widetilde{R}+\sum_{i=1}^{j} E_{i} \in W_{r}^{n+r j}(F, k+j)$.

Evidently it suffices to prove this for $j=1$, or with $E=E_{1}$ that $\widetilde{R}+E \in W_{r}^{n+r}(k+1)$. Let $\widetilde{R}+E=\sum_{i=1}^{m} S_{i}, S_{i} \in G_{r}^{n+r}$, and denote by $S_{i}^{\prime}$ the projection of $S_{i}$ on $V_{r}^{n}$. Then $S_{i}^{\prime}$ is simple and $\widetilde{R}=\sum_{i=1}^{m} S_{i}^{\prime}$. Therefore, $\widetilde{R} \in W_{r}^{n}(k)$ implies $m \geqq k$ and that for $m=k$ all $S_{i}^{\prime} \neq 0$. We show that $m=k$ is impossible.

There are at least two $S_{i}$ which do not lie in $G_{r}^{n}$. For, $S_{i} \in G_{r}^{n}$, if $i>1$, would entail $S_{1}=S_{1}^{\prime}+E$ with $S_{1}^{\prime} \wedge E \neq 0$, but $S_{1}^{\prime}+E$ is not simple by (2.10). Assume that $S_{1}$ and $S_{2}$ do not lie in $G_{r}^{n}$. For $w=\sum_{i=1}^{n+r} \alpha^{i} e_{i}$, put $w^{\prime}=\sum_{i=1}^{n} \alpha^{i} e_{i}$ and $w^{\prime \prime}=\sum_{i=n+1}^{n+r} \alpha^{i} e_{i}$.

Then

$$
S_{i}=\bigwedge_{j=1}^{r} w_{i j}=\bigwedge_{j=1}^{r}\left(w_{i}^{\prime}+w_{j}^{\prime \prime}\right)
$$

and we may assume further that $w_{11}^{\prime \prime} \neq 0$ and $w_{21}^{\prime \prime} \neq 0$.

There are subscripts $i, j, k, l$ with $i \neq k$ such that $w_{i j}^{\prime \prime} \wedge w_{k l}^{\prime \prime} \neq 0$. Otherwise $w_{11}^{\prime \prime} \wedge w_{k l}^{\prime \prime}=0$ for $k \neq 0$ so that $w_{k l}^{\prime \prime}=\lambda_{k l} w_{11}^{\prime \prime}$ for $k \neq 1$. Similarly $w_{k l}^{\prime \prime}=\mu_{k l} w_{21}^{\prime \prime}$ for $k \neq 2$, so that $w_{1 l}^{\prime \prime}=\mu_{1 l} \lambda_{21} w_{11}^{\prime \prime}$.

This, with $\lambda_{11}=1$ and $\lambda_{1 l}=\mu_{1 l} \lambda_{21}$, gives

$$
S_{i}=\bigwedge_{j=1}^{r}\left(w_{j}^{\prime}+\lambda_{i j} w_{11}^{\prime \prime}\right)
$$

But then $\Sigma S_{i}$ cannot produce $E$. Thus we may assume (with a possible change of notation) that $w_{11}^{\prime \prime} \wedge w_{21}^{\prime \prime} \neq 0$. Then $e_{1} \wedge \cdots \wedge e_{n} \wedge w_{11} \wedge w_{21}=$ $e_{1} \wedge \cdots \wedge e_{n} \wedge w_{11}^{\prime \prime} \wedge w_{21}^{\prime \prime} \neq 0$ and there is a base $\left\{e_{i}^{\prime}\right\}$ of $V^{n+r}$ with $e_{i}^{\prime}=e_{i}$ for $i \leqq n, e_{n+1}^{\prime}=w_{11}$, and $e_{n+2}^{\prime}=w_{21}$. Then with the original $\hat{R}$, $E, S_{1}, \cdots, S_{k}$,

$$
(\widetilde{R}+E) \wedge e_{n+1}^{\prime} \wedge e_{n+2}^{\prime}=\left(S_{3}+\cdots+S_{k}\right) \wedge e_{n+1}^{\prime} \wedge e_{n+2}^{\prime},
$$

i.e.,

$$
\widetilde{R} \wedge e_{n+1}^{\prime} \wedge e_{n+2}^{\prime} \in W_{r+2}^{n+r}(k-1)
$$

contradicting (2.2) and (2.7),

3. The sets $V_{r}^{n}\left(F_{t}, k\right)$. Let $F_{t}$ be a topological field. Obviously $G_{r}^{n}\left(F_{t}\right)=V_{r}^{n}\left(F_{t}, 1\right)=W_{r}^{n}\left(F_{t}, 1\right)$ is a closed set in $V_{r}^{n}\left(F_{t}\right)$. It is clear that for $k<N\left(F_{t}, n, r\right)$ the set $V_{r}^{n}\left(F_{t}, k\right)$ cannot be open, but one might expect it to be closed. This is true for $r=2$, see below, but in general not for $r>2$. To show the latter it is not necessary to study general $n$ and $r>2$ because of the following:

Theorem 3.1. If for a topological field $F_{t}$ the set $V_{r}^{n}\left(F_{t}, k\right)$ is 
not closed in $V_{r}^{n}\left(F_{t}\right)$ then for $m \geqq n, s \geqq r, m-s \geqq n-r$ and $j \geqq 0$ the set $V_{s}^{m+j s}\left(F_{t}, k+j\right)$ is not closed in $V_{s}^{m+j s}\left(F_{t}\right)$.

First let $j=0, m \geqq n$ and $\widetilde{R} \in V_{r}^{n}\left(F_{t}, k\right)$. By (2.7) $\widetilde{R} \in V_{r}^{m}\left(F_{t}, k\right)$ so that the latter is not closed. For any $m$ we conclude from $\widetilde{R} \in V_{r}^{m}\left(F_{t}, k\right)$ and (2.2) that

$$
\widetilde{R} \wedge e_{m+1} \wedge \cdots \wedge e_{m+h} \in V_{r+k}^{m+k}\left(F_{t}, k\right) .
$$

Since $V_{r}^{m}\left(F_{t}, k\right)$ is not closed there are $\widetilde{R}_{\nu}$ in $V_{r}^{m}\left(F_{t}, k\right)(\nu=1,2, \cdots)$ such that $\widetilde{R}_{\nu} \rightarrow \widetilde{R} \in W_{r}^{m}\left(F_{t}, k^{\prime}\right)$ with $k^{\prime}>k$.

Then by $(2.2)$

$$
\widetilde{R}_{\nu} \wedge e_{m+1} \wedge \cdots \wedge e_{m+h} \longrightarrow \widetilde{R} \wedge e_{m+1} \wedge \cdots \wedge e_{m+h} \in W_{r+h}^{m+h}\left(F_{t}, k^{\prime}\right)
$$

so that $V_{r+h}^{m+h}\left(F_{t}, k\right)$ is not closed. This settles the case $j=0$ or that $V_{s}^{m}\left(F_{t}, k\right)$ is not closed.

With the notation of (2.15) we see with the same argument

$$
V_{s}^{m+j s}\left(F_{t}, k+j\right) \ni \widehat{R}_{\imath}+\sum_{i=1}^{j} E_{i} \longrightarrow \widetilde{R}+\sum_{i=1}^{j} E_{i} \in W_{s}^{m+j s}\left(F_{t}, k^{\prime}+j\right)
$$

which proves (3.1).

In $\S 5$ it will be shown that $N(F, 6,3)=3$ and $V_{3}^{6}(\boldsymbol{R}$ resp. $C$, 2) is not closed in $V_{3}^{6}(\boldsymbol{R}$ resp. $\boldsymbol{C})$. Probably no $V_{r}^{n}(\boldsymbol{R}$ resp. $\boldsymbol{C}, k)$ with $3 \leqq r \leqq n-3$ and $1<k<N(\boldsymbol{R}$ resp. $\boldsymbol{C}, n, r)$ is closed, but from (3.1) we obtain (with $2+j=k$ ) this best possible result only for $k=2$.

THEOREM 3.2. The sets $V_{r}^{n}(\boldsymbol{R}, k)$ and $V_{r}^{n}(\boldsymbol{C}, k)$ are not closed in $V_{r}^{n}(\boldsymbol{R}) \operatorname{resp} . V_{r}^{n}(\boldsymbol{C})$ when $k \geqq 2, r \geqq 3$, and $n \geqq(k-1) r+3$.

The mentioned best result would require a direct treatment of the case $k>2$ instead of reduction to $k=2$. The fact that we use Plücker relations in $\$ 5$, which become very involved for large $n, r, k$, is responsible for our incomplete result in the case $k>2$.

We now discuss the case $r=2$. The by (2.11) necessary and sufficient condition $\widetilde{R}^{(k+1)}=0$ for $\tilde{R} \in V_{2}^{n}(F, k)$ amounts to polynomial conditions on the components $\alpha^{i k}$ of $\widetilde{R}=\sum_{1 \leqq i<k \leqq n} \alpha^{i k} e_{i} \wedge e_{k}$. The set $V_{2}^{n}(F, k)$ is therefore an algebraic cone in $V_{2}^{n}(F)$ and hence closed when $F$ carries a topology.

It is also clear that for $1 \leqq k<k^{\prime} \leqq[n / 2]$ the set $V_{2}^{n}(F, k)$ is a proper subset of $V_{2}^{n}\left(F, k^{\prime}\right)$ and plausible but, since we do not know whether $V_{2}^{n}(F, k)$ is an irreducible manifold, not a priori certain, that the dimension in the sense of algebraic geometry (denoted by a-dim) and consequently in the case of $\boldsymbol{R}$ resp. $\boldsymbol{C}$ also the topological dimen$\operatorname{sion}(=\operatorname{dim})$, of $V_{2}^{n}(F, k)$ is less than that of $V_{2}^{n}\left(F, k^{\prime}\right)$. That a proof is necessary may be seen from the case $r=3$ (see $\S \S 5$ and 6 ). In 
spite of $N(F, 6,3)=3$ the sets $W_{3}^{6}(\boldsymbol{R}$ resp. $\boldsymbol{C}, 2)$ and $W_{3}^{6}(\boldsymbol{R}, 3)$ have nonempty interiors in $V_{3}^{6}(\boldsymbol{R}$ resp. $\boldsymbol{C})$ so that

$$
\begin{aligned}
& \operatorname{dim} V_{3}^{6}(\boldsymbol{R} \text { resp. } \boldsymbol{C}, 2)=\operatorname{dim} V_{3}^{6}(\boldsymbol{R} \text { resp. } \boldsymbol{C}) \\
& \operatorname{dim} W_{3}^{6}(\boldsymbol{R}, 2)=\operatorname{dim} W_{3}^{6}(\boldsymbol{R}, 3)=\operatorname{dim} V_{3}^{6}(\boldsymbol{R})=20 .
\end{aligned}
$$

But $W_{3}^{6}(\boldsymbol{C}, 3)$ has no interior points and hence by a theorem in dimension theory (see [2, p. 46])

$$
\operatorname{dim} W_{3}^{6}(\boldsymbol{C}, 3)<\operatorname{dim} W_{3}^{6}(\boldsymbol{C}, 2)=\operatorname{dim} V_{3}^{6}(\boldsymbol{C})=40 .
$$

Although we need only the expression for $\widetilde{R}^{(k)}$ in the case $r=2$, we give, owing to its potential usefulness, the expression of $\bigwedge_{i=1}^{k} \widetilde{R}_{i}$ of $k$ different r-vectors in terms of the components of the $\widetilde{R}_{i}$. The rather long proof can be found in [1, p. 51].

Put $J=\left\{j_{1}, \cdots, j_{r}\right\}$ where $1 \leqq j_{1}<\cdots<j_{r} \leqq n, n \geqq k r$.

Let $\alpha_{i}^{J}=\alpha_{i}^{j_{1} \cdots j_{r}}(i=1, \cdots, k)$ be indeterminates and define for a permutation $\pi$ of $\{1, \cdots, r\}$

$$
\alpha_{i}^{\pi(J)}=\alpha_{i}^{j_{\pi(1)} \ldots j_{\pi(r)}}=\operatorname{sgn} \pi \alpha_{i}^{I} .
$$

If $\left\{H=h_{1}, \cdots, h_{k r}\right\}$ with $1 \leqq h_{1}<\cdots<h_{k r} \leqq n$ and $J_{1} \cup \cdots \cup J_{k}=H$ (disregarding order) then $J_{\nu} \cap J_{\mu}=\phi$ for $\nu \neq \mu$ and $J_{1}, \cdots, J_{k}$ in this order is a permutation of $H$ whose sign is denoted by

$$
\left[\begin{array}{c}
J_{1} \cdots J_{k} \\
H
\end{array}\right]
$$

We then define

$$
F^{H}\left(\alpha_{1}, \cdots, \alpha_{k}\right)=\sum_{J_{1} \cup \cdots \cup J_{k}=H}\left[\begin{array}{c}
J_{1} \cdots J_{k} \\
H
\end{array}\right] \alpha_{1}^{J_{1}} \cdots \alpha_{k}^{I_{k}}
$$

where $\alpha_{\nu}$ stands for $\left\{\alpha_{\nu}^{J}: J \subset H\right\}$. If $\pi$ is a permutation of $\{1, \cdots, k\}$, then

$$
F^{H}\left(\alpha_{\pi(1)}, \ldots, \alpha_{\pi(k)}\right)=(\operatorname{sgn} \pi)^{r} F^{H}\left(\alpha_{1}, \cdots, \alpha_{k}\right) .
$$

THEOREM 3.3. If $\widetilde{R}_{i}=\sum_{J \subset N} \alpha_{i}^{J} e_{J}$ with $N=\{1, \cdots, n\}$, then $\bigwedge_{i=1}^{k} \widetilde{R}_{i}=\sum_{H \subset N} F^{H}\left(\alpha_{1}, \cdots, \alpha_{k}\right) e_{H}$.

Consequently, if $Q^{H}(\alpha)$ originates from $F^{H}\left(\alpha_{1}, \cdots, \alpha_{k}\right)$ through replacing each $\alpha_{i}$ by the same $\alpha=\left\{\alpha^{J}\right\}$, then we obtain

Corollary 3.4. If $\widetilde{R}=\sum_{J \subset N} \alpha^{J} e_{J}$, then $\widetilde{R}^{(k)}=k ! \sum_{H \subset N} Q^{H}(\alpha) e_{H}$.

From (3.4) one deduces with the conventions $\left(\begin{array}{l}0 \\ 2\end{array}\right)=\left(\begin{array}{l}1 \\ 2\end{array}\right)=0$, 


$$
a-\operatorname{dim} V_{2}^{n}(F, k)=\left(\begin{array}{c}
n \\
2
\end{array}\right)-\left(\begin{array}{c}
n-2 k \\
2
\end{array}\right), 1 \leqq k \leqq\left[\frac{n}{2}\right]
$$

(see [1, p. 65]). Hence

$\operatorname{dim} V_{2}^{n}(\boldsymbol{R}, k)=\left(\begin{array}{l}n \\ 2\end{array}\right)-\left(\begin{array}{c}n-2 k \\ 2\end{array}\right), \operatorname{dim} V_{2}^{n}(\boldsymbol{C}, k)=2\left[\left(\begin{array}{l}n \\ 2\end{array}\right)-\left(\begin{array}{c}n-2 k \\ 2\end{array}\right)\right]$, and so

$\operatorname{dim} V_{2}^{n}(\boldsymbol{R} \operatorname{resp.} \boldsymbol{C}, k)<\operatorname{dim} V_{2}^{n}(\boldsymbol{R}$ resp. $\boldsymbol{C}, k+1)$ for $1 \leqq k<\left[\frac{n}{2}\right]$ in contrast to the case $r=3$.

4. Uniqueness. Let $R_{i} \in G_{r}^{n}(F),(i=1, \cdots, k)$. The sum $\sum_{i=1}^{k} R_{i}$ is called unique in $V_{r}^{n}(F)$ if $S_{i} \in G_{r}^{n}(F)(i=1, \cdots, k)$ and $\Sigma R_{i}=\Sigma S_{i}$ imply that $S_{\pi(i)}=R_{i}(i=1, \cdots, k)$ for a suitable permutation $\pi$ of $\{1, \cdots, k\}$.

Obviously:

(4.1) If $\sum_{i=1}^{k} R_{i}$ is unique then it is irreducible.

(4.2) If $\sum_{i=1}^{k} R_{i}$ is irreducible resp. unique then so is $\sum_{i=1}^{j} R_{i}$ for $j<k$.

The converse of (4.1) does not hold; in particular:

(4.3) If $r=2, k>1, \bigwedge_{i=1}^{k} R_{i} \neq 0$ then $\sum_{i=1}^{k} R_{i}$ is not unique, i.e., no irreducible sum of 2-vectors is unique.

Because of (4.2) it suffices to observe that

$$
e_{1} \wedge e_{2}+e_{3} \wedge e_{4}=\left(e_{1}+e_{3}\right) \wedge e_{2}+e_{3} \wedge\left(-e_{2}+e_{4}\right) \text {. }
$$

However, if $r>2$ and $\bigwedge_{i=1}^{k} R_{i} \neq 0$, then $\sum_{i=1}^{k} R_{i}$ probably is unique. Because the Plücker relations are hard to handle for large $k$ we were able to prove only:

THEOREM 4.4. If $r>2, k \leqq r$, and $\bigwedge_{i=1}^{k} R_{i} \neq 0$, then $\sum_{i=1}^{k} R_{i}$ is unique.

Here both the field $F$ and the dimension $n$ of the space (except that $n \geqq r k$ is, of course, implied) are deliberately omitted because they are immaterial.

First we convince ourselves that $n$ is unimportant and at the end 
of the proof we indicate why $F$ is.

Let $R_{i}=v_{(i-1) r+1} \wedge \cdots \wedge v_{i r}(i=1, \cdots, k), \Lambda v_{i} \neq 0$. It suffices to prove (for a given $F$ ) that $\Sigma R_{i}$ is unique in the space $V$ spanned by $v_{1}, \cdots, v_{k r}$. For, let also

$$
\widetilde{R}=\sum_{i=1}^{k} R_{i}=\Sigma R_{i}^{*}, R_{i}^{*} \in V_{r}^{n}, V^{n} \supset V .
$$

Under projection of $V^{n}$ on $V$ let $R_{i}^{*} \rightarrow R_{i}^{\prime}$. Then $\Sigma R_{i}^{*} \rightarrow \Sigma R_{i}^{\prime}, \widetilde{R} \rightarrow \widetilde{R}$, $\Lambda R_{i}^{*} \rightarrow \Lambda R_{i}^{\prime}$ so that $\widetilde{R}=\Sigma R_{i}^{\prime}$ and if (4.4) holds in $V$ then $\left\{R_{i}^{\prime}\right\}$ is a permutation of $\left\{R_{i}\right\}$. Therefore, $\Lambda R_{i}^{\prime} \neq 0$ and hence $\Lambda R_{i}^{*} \neq 0$. If $R_{i}^{*}=v_{(i-1) r+1}^{*} \wedge \cdots \wedge v_{i r}^{*}$ then because (4.4) holds in the space spanned by $v_{1}^{*}, \cdots, v_{r k}^{*}$ we have $R_{\pi(i)}^{*}=R_{i}$ for a suitable permutation $\pi$ of $\{i, \cdots, k\}$.

In the proof of (4.4) we therefore assume that $n=r k$ and $\widetilde{R}=$ $\sum_{i=1}^{k} R_{i}$ with

$$
R_{i}=e_{(i-1) r+1} \wedge \cdots \wedge e_{i r}=e_{L(i)}
$$

where

$$
L(\nu)=\{(\nu-1) r+1, \cdots, \nu r\} \quad(\nu=1, \cdots, k) .
$$

We further put

$$
I=\left\{i_{1}, \cdots, i_{k}\right\} \quad \text { with } 1 \leqq i_{1}<\cdots<i_{k} \leqq r k,
$$

also

$$
I(\nu)=I /\left\{i_{\nu}\right\}, I(\nu, \mu)=I /\left\{i_{\nu}, i_{\mu}\right\} \text {, etc . }
$$

It will prove convenient and causes no ambiguities to use $I(\nu)$ for $\left\{i_{1}, \cdots, i_{\nu-1}, i_{\nu+1}, \cdots, i_{k}\right\}$ even if $i_{\nu}$ is not defined.

$\Omega$ is the set of all $I$ with $i_{\nu} \in L(\nu)(\nu=1, \cdots, k)$, and $I(\nu) \in \Omega$ means $i_{\mu} \in L(\mu)$ for $\mu \neq \nu$.

We also use

$$
e_{L(\nu)}=\bigwedge_{i \in L(v)} e_{i}, \quad e_{I}=\bigwedge_{i \in I} e_{i}, \text { etc. }
$$

The sign depends on the order but will prove irrelevant. Finally $E(\nu)$ and $F(\nu)$ are the spaces spanned by the $e_{i}$ with $i \in L(\nu)$ or $i \notin$ $L(\nu)$ respectively.

From now on we will often use the Plücker relations (see [3, p. 23] and [4, p. 27]) which in our type of notation may be stated as follows:

Let $P=\left\{p_{1}, \cdots, p_{r}\right\}, 1 \leqq p_{i} \leqq n, P(i)=P /\left\{p_{i}\right\}$,

$$
\alpha^{P}=\alpha^{p_{1} \cdots p_{r}}=\operatorname{sgn} \pi \alpha^{p_{\pi(1)} \cdots p_{\pi(r)}}
$$

for a permutation $\pi$ of $\{1, \cdots, r\}$ and similarly for $Q$. The vector 


$$
\frac{1}{r !} \sum_{P} \alpha^{P} e_{P}=\sum_{1 \leqq p_{1}<\cdots<p_{r} \leqq n} \alpha^{p_{1} \cdots p_{r}} e_{p_{1}} \wedge \cdots \wedge e_{p_{r}} \in V_{r}^{n}
$$

is simple if and only if for any $P, Q$

Plïcker: $\quad \alpha^{P} \alpha^{Q}+\sum_{i=1}^{r}(-1)^{i} \alpha^{P(r) q_{i}} \alpha^{p} r^{Q(i)}=0$.

We prove several lemmas beginning with

(4.5) Let $\widetilde{T}=\sum \gamma^{I} e_{I}$ and suppose $\widetilde{T} \wedge \widetilde{R}=0$. If $k<r$, or $k=$ $r$ but $\widetilde{T}$ is simple, then $\gamma^{I} \neq 0$ only if $I \in \Omega$. Thus simple $\widetilde{T} \neq 0$ implies $\gamma^{I} \neq 0$ for at least one $I \in \Omega$.

If $k<r$ the assertion follows from

$$
\begin{aligned}
& \widetilde{R} \wedge \widetilde{T}=\sum_{\nu=1}^{k}\left[e_{L(\nu)} \wedge\left(\sum_{I \cap L(\nu)=\phi} \gamma^{I} e_{I}\right)\right], \\
& e_{L(\nu)} \wedge e_{I} \neq e_{L(\mu)} \wedge e_{I} \text { for } \nu \neq \mu \text { and } k<r \text {, }
\end{aligned}
$$

and the observation that $I \cap L(\nu)=\phi$ for some $\nu$ is equivalent to $I \notin \Omega$.

If $k=r$ then the terms in $\widetilde{R} \wedge \widetilde{T}$ with $e_{L(1)}$ as a factor are

$$
e_{L(1)} \wedge\left[\sum_{i_{1}>r} \gamma^{I} e_{I}+(-1)^{r} \gamma^{L(1)}\left(e_{L(2)}+\cdots+e_{L(k)}\right)\right] \text {. }
$$

Therefore, $\gamma^{I}=0$ if $i_{1}>r$ (hence $I \notin \Omega$ ) and $\gamma^{L(\nu)}+(-1)^{r} \gamma^{L(1)}=0$ for $\nu>1$. Generally $\gamma^{I}=0$ if, $I \notin \Omega$ and $I$ is no $L(\nu)$; moreover,

$$
\gamma^{L(\mu)}+(-1)^{r} \gamma^{L(\nu)}=0 \text { if } \mu \neq \nu .
$$

If $r$ is even then $\gamma^{L(\nu)}=0$ for all $\nu$ so that $\gamma^{I} \neq 0$ only for $I \in \Omega$.

If $r$ is odd then $\gamma^{L(\nu)}=\lambda$ for all $\nu$ and thus

$$
\widetilde{T}=\lambda \widetilde{R}+\sum_{I \in \Omega} \gamma^{I} e_{I}
$$

We show $\widetilde{T}$ is simple only if $\lambda=0$ which completes the assertion.

Let $I \in \Omega$ and assume $i_{1} \neq r$. Then with

$$
L(1, r) i_{s}=\left\{1, \cdots, r-1, i_{s}\right\}
$$

one of the Plücker relations for the simplicity of $\widetilde{T}$ is

$$
0=\gamma^{L(1)} \gamma^{I}+\sum_{s=1}^{r}(-1)^{s} \gamma^{L(1, r) i_{s}} \gamma^{r I(s)}=\lambda \gamma^{I},
$$

for $\gamma^{L(1, r) i_{s}}=0$ because $L(1, r) \in \bigcup_{\nu=1}^{r} L(\nu) \cup \Omega$ for $s>1$, and $L(1, r) i_{1}$ contains a repeated index (since $i_{1} \neq r$ ). If $i_{1}=r$ just permute $L(1)$ so that $r$ is not the last element. Thus $\gamma^{I}=0$ for all $I \in \Omega$, or 
$\lambda=0$. Since $\widetilde{T}=\lambda \widetilde{R}$ would not be simple we must have $\lambda=0$.

Let $H=\left\{h_{1}, \cdots, h_{k-1}\right\}$ with $1 \leqq h_{1}<\cdots<h_{k-1} \leqq r k$.

(4.6) If $\widetilde{S}=\sum \beta^{H} e_{H}$ and $\widetilde{R} \wedge \widetilde{S}$ is simple then, for

$$
I \in \Omega, \beta^{I(s)} \beta^{I(t)}=0
$$

if $s \neq t$.

The terms in the expansion

$$
\widetilde{R} \wedge \widetilde{S}=\Sigma \alpha^{i_{1} \cdots i_{r+k-1}} e_{i \cdots i_{r+k-1}}
$$

which contain $e_{I}$ as a factor are given by

$$
e_{I} \wedge\left[ \pm \beta^{I(1)} e_{L\left(1, i_{1}\right)} \pm \cdots \pm \beta^{I(k)} e_{L\left(k, i_{k}\right)}\right]
$$

where $L\left(\nu, i_{\nu}\right)=L(\nu) /\left\{i_{\nu}\right\}$. Consider the Plücker relation for $\widetilde{R} \wedge \widetilde{S}$ beginning with

$$
\alpha^{I, L\left(s, i_{s}\right)} \alpha^{I, L\left(t, i_{t}\right)}=\beta^{I(s)} \beta^{I(t)} \text {. }
$$

The terms not written down all vanish. The first $k-1$ that follow vanish because the first factor has a repeated superscript. From the $(k+1)$ st term on, the last element of $L\left(i, i_{s}\right)$ is the first superscript of the second factor $\alpha$ which then vanishes because it does not appear in (4.7). (This requires $r \geqq 3$. The first $\alpha$ also vanishes and for a similar reason.)

The following is the decisive step in our long argument:

(4.8) If both $S=\Sigma \beta^{H} e_{H}$ and $\widetilde{R} \wedge S$ are simple and some $\beta^{I(1)} \neq 0$ $(I(1) \in \Omega)$ then $\beta^{i_{1} \nu_{1} \cdots \nu_{k-2}}=0$ for $i_{1} \in L(1)$ and any $\nu_{s}(s=1, \cdots, k-2)$. Briefly $S \in F(1)_{k-1}$.

Take any $i_{1} \in L(1)$ and join it to $I(1)$. This produces an $I \in \Omega$. We prove inductively.

$$
\beta^{\nu_{1} \cdots \nu_{\lambda} I(k-\lambda, \cdots, k)}=0 \text { for all } \nu_{s} \text { and } \lambda \leqq k-2 .
$$

If $k=2$ we have $\beta^{i_{1}}=\beta^{I(2)}=0$ by (4.6) and $\beta^{I(1)} \neq 0$.

If $k \geqq 3$ we make

Step 1. Consider the Plücker relation

$$
\begin{aligned}
& 0=\beta^{I(1)} \beta^{\nu I(k-1, k)}-\beta^{I(1, k) \nu} \beta^{i_{k} I(k-1, k)}+\beta^{I(1, k) i_{1}} \beta^{i_{k} \nu I(1, k-1, k)} \\
& -\beta^{I(1, k) i_{2}} \beta^{i_{k}^{\nu L(2, k-1, k)}} \pm \cdots \pm \beta^{I(1, k) i_{k-2}} \beta^{i_{k}^{\nu L(k-2, k-1, k)}} \text {. }
\end{aligned}
$$

Except for order 


$$
i_{k} I(k-1, k)=I(k-1), \quad \text { and } I(1, k) i_{1}=I(k)
$$

so that the second and third terms vanish by (4.6). The remaining terms vanish because the sets $I(1, k) i_{2}, \cdots, I(1, k) i_{k-2}$ contain repeated elements. If $k=3$ we are finished. If $k>3$ we make

Step 2. Take the Plücker relation

$$
\begin{aligned}
0= & \beta^{I(1)} \beta^{\nu \mu I(k-2, k-1, k)}-\beta^{I(1, k) \nu} \beta^{i_{k} \mu I(k-2, k-1, k)} \\
& +\beta^{I(1, k) \mu} \beta^{i_{k} \nu I(k-2, k-1, k)}-\beta^{I(1, k) i_{1}} \beta^{i_{k} \nu \mu I(1, k-2, k-1, k)} \\
& +\beta^{I(1, k) i_{2}} \beta^{i_{k}^{\nu} \mu I(2, k-2, k-1, k)}-\cdots \pm \beta^{I(1, k) i_{k-3}} \beta^{i_{k} \nu \mu I(k-3, k-2, k-1, k)} .
\end{aligned}
$$

Except for order

$$
i_{k} \mu I(k-2, k-1, k)=\mu I(k-2, k-1)
$$

and

$$
i_{k} \nu I(k-2, k-1, k)=\nu I(k-2, k-1)
$$

so that the second and third terms vanish by Step 1 (that $k-1, k$ are replaced by $k-2, k-1$ is immaterial since the argument of Step 1 is the same for any permutation of $\{2, \cdots, k\})$. The fourth term vanishes because of $(4.6)$ and $I(1, k) i_{1}=I(k)$. In all following terms the sets of superscripts in the first factor $\beta$ contain repeated elements and these terms vanish also. This completes the argument in case $k=4$. If $k>4$, the process clearly continues.

(4.9) If both $S=\Sigma \beta^{H} e_{H}$ and $\widetilde{R} \wedge S$ are simple and $\beta^{I(t)} \neq 0$ for some $I(t) \in \Omega$ then $S \in F(t)_{k-1}$. If $S=w_{1} \wedge \cdots \wedge w_{k-1}$ then each $w_{i} \in$ $F(t)$.

The first part is a consequence of (4.8). The second statement follows from the general lemma.

(4.10) If $m<n, V^{m} \subset V^{n}, v_{j} \in V^{n}, \bigwedge_{j=1}^{s} v_{j} \neq 0$ and $\bigwedge_{j=1}^{s} v_{j} \in V_{s}^{m}$ then $v_{\jmath} \in V^{m}(j=1, \cdots s)$.

Setting $v_{i}=v_{i}^{\prime}+\beta_{i} e_{n}(i=1, \cdots, s)$ in the proof of (2.1) yields the case $m=n-1$ from which the general case follows.

Theorem 4.11. If $k \leqq r, S=\bigwedge_{i=1}^{k-1} w_{i} \neq 0$ and $\widetilde{R} \wedge S$ is simple then $w_{i} \in F(t)$ for a suitable $t$.

If $S=\Sigma \beta^{H} e_{H}$ then it suffices by (4.9) to show that $\beta^{I(t)} \neq 0$ for a suitable $I(t) \in \Omega$. Because $\widetilde{R} \wedge S$ is a simple $(r+k-1)$-vector there is a vector $v=\sum_{i=1}^{r k} \delta^{i} e_{i}$ such that $\widetilde{R} \wedge S \wedge v=0$ and $S \wedge v \neq 0$. If 
$T=S \wedge v=\Sigma \gamma^{J} e^{J}$ then

$$
\gamma^{J}=\Sigma \pm \beta^{J(t)} \delta^{j t}
$$

By (4.5) there is at least one $I \in \Omega$ with $\gamma^{I} \neq 0$, hence $\beta^{I(t)} \neq 0$ for some $t$.

After these preparations we are ready to prove (4.4). First observe

$$
\text { If } \widetilde{R}=\sum_{\nu=1}^{k} v_{L(\nu)}\left(v_{i} \in V\right) \text { then } \bigwedge_{i=1}^{r k} v_{i} \neq 0 \text {. }
$$

Each $v_{i} \neq 0$ because $\sum_{i=1}^{k} e_{L(\nu)}=\widetilde{R}$ is irreducible. Assme $\Lambda v_{i}=0$ and let $\left\{w_{j}\right\}, 1 \leqq j \leqq \lambda<k r$, be a maximal set of independent $v_{i}$. Since the $w_{i}$ span a proper subspace $V^{\prime}$ of $V$, an $e_{\mu}$ with $w_{1} \wedge \cdots \wedge$ $w_{\lambda} \wedge e_{\mu} \neq 0$ exists, and the $w_{i}$ together with $e_{\mu}$ span a space $V^{\prime \prime}$ with $V^{\prime} \subset V^{\prime \prime} \subset V$. Now $\Sigma v_{L(\nu)}$ is irreducible in $V$ and $V^{\prime}$, and therefore (see (2.2) and (2.7)) $\Sigma_{L(\nu)} \wedge e_{\mu}$ is irreducible in $V^{\prime \prime}$ and in $V$. But if $\mu \in L(s)$ then $\widetilde{R} \wedge e_{\mu}=\sum_{\nu \neq s} e_{L(\nu)} \wedge e_{\mu}$.

(4.13) If $\tilde{R}=\sum_{\nu=1}^{k} v_{L(\nu)}\left(v_{i} \in V\right), k \leqq r, I=\left\{i_{1}, \cdots, i_{k}\right\} \in \Omega$ then $v_{i_{t}} \in E(\pi(t))$ for a suitable permutation $\pi$ of $\{1, \cdots, k\}$.

First $v_{I(s)} \neq 0$ by (4.12). Next

$$
\widetilde{R} \wedge v_{I(s)}=v_{L(s)} \wedge v_{I(s)} \neq 0
$$

is simple. Therefore (4.11) yields $v_{i_{\nu}} \in F(\pi(s)),(\boldsymbol{\nu} \neq s)$ for a suitable number $\pi(s),(1 \leqq \pi(s) \leqq k)$. We must show that $\pi(s)$ defines a permutation of $\{1, \cdots, k\}$ or that $\pi(s) \neq \pi(t)$ for $s \neq t$. Assume $\pi(s)=\pi(t)$ for some $s \neq t$. Then $v_{i_{\nu}} \in F(\pi(s))$ for $\nu=1, \cdots, k$ because $I(s) \cup I(t)=$ $I$ whence $v_{I} \in F(\pi(s))_{k}$ and $\widetilde{R} \wedge v_{I}=e_{L(\pi(s))} \wedge v_{I} \neq 0$ contradicting $\widetilde{R} \wedge$ $v_{I}=0$. Thus $v_{i_{t}} \in \bigcap_{i_{t} \in I(s)} F(\pi(s))=\bigcap_{s \neq t} F(\pi(s))=E(\pi(t))$.

This establishes the uniqueness of $\Sigma e_{L(\nu)}$. For, consider $I \in \Omega$ and put $I^{\prime}=\left\{j_{1}, i_{2}, \cdots, i_{k}\right\}$ with $j_{1} \in L(1)$. Then $I^{\prime} \in \Omega$. Since $v_{i_{\nu}} \in E(\pi(\nu))$ for $\nu>1$ it follows from (4.13) that $v_{j_{1}} \in E(\pi(1))$. Thus $v_{j_{1}} \in E(\pi(1))$ for all $j_{1} \in L(1)$ and $v_{L(1)}=\alpha_{1} e_{L(\pi(1))}$.

Generally, $v_{L(\nu)}=\alpha_{\nu} e_{L(\pi(\nu))}$ whence $\alpha_{\nu}=1(\nu=1, \cdots, k)$ and uniqueness follows.

The condition $v_{i} \in V$ which entered the proof of (4.12) because we applied (2.2) can now be eliminated; $\Sigma e_{\left.L^{\prime} \nu\right)}$ retains its form after extension of the underlying field and therefore remains unique after the extension. This justifies the formulation of (4.4) which does not mention a field.

5. The case $n=6, r=3$. The remainder of the paper deals with the case $n=6, r=3$ whose importance was noted in connection 
with (3.2). We first show $N(F, 6,3)=3$ which may be new for $F \neq$ C. Our inequalities (2.3) and (2.5) give only

$$
2=N(F, 5,2) \leqq N(F, 6,3) \leqq N(F, 5,3)+N(F, 5,2)=4 .
$$

With $e_{i j k}=e_{i} \wedge e_{j} \wedge e_{k}$ we prove:

(5.1) $\widetilde{S}=e_{145}+e_{246}+e_{356}$ is irreducible; whence $N(F, 6,3) \geqq 3$.

This proof rests on the observation:

$$
\text { If } R=\widetilde{S} \wedge \sum_{i=1}^{6} \beta^{i} e_{i} \text { is simple then } \beta^{1}=\beta^{2}=\beta^{3}=0 .
$$

(The converse is trivial but not needed.) If

$$
R=\sum_{1 \geqq i<j<k<l<\geqq 6} \alpha^{i j k l} e_{i j k l}
$$

then

$$
\sum_{1<j<k<6} \alpha^{1 j k 6} e_{1 j k 6}=e_{16} \wedge\left[\beta^{6} e_{45}-\beta^{1}\left(e_{24}+e_{35}\right)\right] .
$$

Therefore one of the Plücker relations for $R$ is

$$
0=\alpha^{1624} \alpha^{1635}-\alpha^{1623} \alpha^{4165}+\alpha^{1625} \alpha^{4613}=\left(\beta^{1}\right)^{2} .
$$

Similarly, $\beta^{2}=\beta^{3}=0$.

Assume $\widetilde{S}$ were reducible, $\widetilde{S}=v_{123}+v_{456}$ (where again $v_{i j k}=v_{i} \wedge$ $v_{j} \wedge v_{k}$ ) with $v_{i}=\sum_{k=1}^{6} \beta_{i}^{k} e_{k}$. Then $\widetilde{S} \wedge v_{i}$ is simple, so that by (5.2) $\beta_{i}^{k}=0$ for $k \leqq 3$, whence

$$
\widetilde{S}=\left[\operatorname{det}\left(\beta_{k}^{3+i}\right)+\operatorname{det}\left(\beta_{3+k}^{3+i}\right)\right] e_{456},
$$

which is false because $\widetilde{S} \wedge e_{6}=e_{1456} \neq 0$.

To show $N(F, 6,3) \leqq 3$ we need the lemma:

(5.3) Given $\widetilde{R}_{i} \in V_{2}^{4}(F)(i=1, \cdots, m)$ there are $\lambda_{i} \in F$ and $R_{i} \in$ $G_{2}^{4}(F)(i=0, \cdots m)$ such that $\widetilde{R}_{i}=R_{i}+\lambda_{i} R_{0}(i=1, \cdots, m)$.

If $\widetilde{R}_{i}$ is simple then $R_{i}=\widetilde{R}_{i}, \lambda_{i}=0$ will do, so we assume that no $\widetilde{R}_{i}$ is simple. $G_{2}^{4}$ is a quadratic cone and a hypersurface in $V_{2}^{4}(F)$. Therefore, $R_{0} \in G_{2}^{4}$ exists such that the tangent hyperplane of $G_{2}^{4}$ at $R_{0}$ does not contain any $\widetilde{R}_{i}$, and no line through $\widetilde{R}_{i}$ and $R_{0}$ intersects $G_{2}^{4}$ (as a locus in $V_{2}^{4}$ completed to a projective space) at infinity. Then the line through $\widetilde{R}_{i}$ and $R_{0}$ intersects $G_{2}^{4}$ in a second point $R_{i}^{\prime}$ so that

$$
\widetilde{R}_{i}=\left(1-\lambda_{i}\right) R_{i}^{\prime}+\lambda_{i} R_{0}=R_{i}+\lambda_{i} R_{0} .
$$

This argument does not require extending $F$ because it amounts to solving a quadratic equation of which one root is $F$. 
Now let $\widetilde{R}=\sum_{1 \leqq i<j<k \leqq 6} \alpha^{i j k} e_{i j k} \in V_{3}^{6}\left(F^{\prime}\right)$ be given. A simple calculation shows that either $\widetilde{R} \in V_{3}^{6}(F, 2)$ or a base $\left\{\bar{e}_{i}\right\}$ exists in terms of which

$$
\widetilde{R}=\sum_{1 \leqq i<j \leqq 4} \beta^{i j 5} \bar{e}_{i j 5}+\sum_{1 \leqq i<j \leqq 4} \beta^{i j 6} \bar{e}_{i j 6}=\widetilde{S}_{1} \wedge \bar{e}_{5}+\widetilde{S}_{2} \wedge \bar{e}_{6}
$$

with $\widetilde{S}_{i} \in V_{2}^{4}$. By (5.3) there are $S_{i} \in G_{2}^{4}$ and $\lambda_{i} \in F$ such that

$$
\begin{aligned}
\widetilde{R} & =\left(S_{1}+\lambda_{1} S_{0}\right) \wedge \bar{e}_{5}+\left(S_{2}+\lambda_{2} S_{0}\right) \wedge \bar{e}_{6} \\
& =S_{1} \wedge \bar{e}_{5}+S_{2} \wedge \bar{e}_{6}+S_{0} \wedge\left(\lambda_{1} \bar{e}_{5}+\lambda_{2} \bar{e}_{6}\right) \in V_{3}^{6}(F, 3) .
\end{aligned}
$$

Thus:

$$
N(F, 6,3)=3 .
$$

By a similar argument we prove

$$
3 \leqq N(F, 7,3) \leqq 5 \text {. }
$$

The left inequality follows from $3=N(6,3) \leqq N(7,3)$, see (2.8). For the right inequality one shows (see $[1$, p. 90]) that either $\widetilde{R} \in$ $V_{3}^{7}(2)$ or with a suitable base $\left\{\bar{e}_{i}\right\}$

$$
\widetilde{R}=\sum_{1 \leqq i<j \leqq 4} \beta^{i j 5} \bar{e}_{i j 5}+\sum_{1 \leqq i<j \leqq 4} \beta^{i j 6} \bar{e}_{i j 6}+\sum_{1 \leqq i<j \leqq 4} \beta^{i j 7} \bar{e}_{i j 7}+\sum_{i=1}^{5} \beta^{i 67} \bar{e}_{i 67} .
$$

The last sum is simple and applying (5.3) to the first three terms on the right yields $N(F, 7,3) \leqq 5$. This method does not extend to $N(n$, 3) with $n>7$.

We now study a special type of $\widetilde{R} \in V_{3}^{8}(\boldsymbol{C})$ which will confirm some of the important assertions made previously.

Let $Y$ be the set of triples

$$
Y=\{123,126,135,156,234,246,345,456\}
$$

and suppose that the $\alpha^{I}, I \in Y$, satisfy the inequalities

$$
\begin{array}{ll}
\alpha^{123} \alpha^{156}+\alpha^{126} \alpha^{135} \neq 0, & \alpha^{123} \alpha^{246}+\alpha^{126} \alpha^{234} \neq 0, \\
\alpha^{123} \alpha^{345}+\alpha^{135} \alpha^{234} \neq 0, & \alpha^{234} \alpha^{456}+\alpha^{246} \alpha^{345} \neq 0, \\
\alpha^{135} \alpha^{456}+\alpha^{156} \alpha^{345} \neq 0, & \alpha^{126} \alpha^{456}+\alpha^{156} \alpha^{246} \neq 0,
\end{array}
$$

and that the roots $\lambda, \mu$ of

$$
\left(\alpha^{123} x-\alpha^{234}\right)\left(\alpha^{156} x-\alpha^{456}\right)+\left(\alpha^{126} x+\alpha^{246}\right)\left(\alpha^{135} x+\alpha^{345}\right)=0
$$

are distinct. They are different from zero.

THEOREM 5.8. If $\widetilde{R}=\sum_{I \in Y} \alpha^{I} e_{I} \in V_{3}^{6}(C)$ and the $\alpha^{1}$ satisfy (5.6), then $\widetilde{R}=R_{\beta}+R_{\gamma}, R_{\beta}=\sum_{I \in Y} \beta^{I} e_{I}, R_{\gamma}=\sum_{I \in Y} \gamma^{I} e_{I}$, where $R_{\beta}$ and $R_{\gamma}$ are simple with $R_{\beta} \wedge R_{\gamma} \neq 0$. Hence the representation $R_{\beta}+R_{\gamma}$ is 
unique (by (4.4)).

If $\lambda, \mu$ are the solutions of (5.7) then the $\beta^{I}$ and $\gamma^{I}(I \in Y)$ are given by

$$
\begin{array}{ll}
\beta^{1 i j}=\frac{\mu \alpha^{1 i j}-\alpha^{4 i j}}{\mu-\lambda}, & \gamma^{1 i j}=\frac{\alpha^{4 i j}-\lambda \alpha^{1 i j}}{\mu-\lambda}, \\
\beta^{4 i j}=\lambda \beta^{1 i j}, & \gamma^{4 i j}=\mu \gamma^{1 i j} .
\end{array}
$$

No $\beta^{I}$ or $\gamma^{I}(I \in Y)$ vanishes.

This representation was found by using Plücker relations (see [1, pp. 98-106]), but after it is explicitly given one readily verifies that $R_{\beta}$ and $R_{r}$ are simple and that $R_{\beta} \wedge R_{\gamma} \neq 0$. In fact, it is easy to factor $R_{\beta}$ and $R_{\gamma}$, see [4, p. 21]: Since $\beta^{I} \neq 0$ if $I \in Y$, letting $\nu=$ $\left(\beta^{123}\right)^{-2 / 3}$ we find

$$
R_{\beta}=u \wedge v \wedge w=\Sigma u^{i} e_{i} \wedge \Sigma v^{i} e_{i} \wedge \Sigma w^{i} e_{i}
$$

with

$$
u^{i}=\nu \beta^{23 i}, v^{i}=-\nu \beta^{13 i}, w^{i}=\nu \beta^{12 i}
$$

(see also [1, p. 102]), and similarly for $R_{r}$.

First we confirm the statement in the introduction that irreducibility may depend on the field.

(5.9) If the $\alpha^{I}$ in (5.8) are real and $\lambda, \mu$ are not, then $\widetilde{R} \in W_{3}^{6}(C, 2)$ but $\widetilde{R} \in W_{3}^{6}(\boldsymbol{R}, 3)$.

For because $R_{\beta}+R_{\gamma}$ is unique, $\widetilde{R} \in V_{3}^{6}(R, 2)$ is impossible, and this with $N(\boldsymbol{R}, 6,3)=3$ entails the assertion.

Next we observe that the vector

$$
\begin{aligned}
\widetilde{R}(\eta)= & e_{1} \wedge\left(e_{2}+e_{5}\right) \wedge e_{6}+e_{1} \wedge e_{3} \wedge e_{5}+\eta e_{2} \wedge e_{4} \wedge e_{6} \\
& +\left(e_{2}+e_{5}\right) \wedge e_{3} \wedge e_{4} \quad(\eta \neq 0)
\end{aligned}
$$

is a special case of (5.8) and that $\lambda, \mu$ are real when $\eta<0$. Letting $\eta \rightarrow 0^{-}$we find

$$
\widetilde{R}\left(0^{-}\right)=e_{1} \wedge\left(e_{2}+e_{5}\right) \wedge e_{6}+e_{1} \wedge e_{3} \wedge e_{5}+\left(e_{2}+e_{5}\right) \wedge e_{3} \wedge e_{4}
$$

which by (5.1) lies in $W_{3}^{6}(\boldsymbol{R}$ or $\boldsymbol{C}, 3)$. Therefore:

(5.10) The sets $V_{3}^{6}(\boldsymbol{R}, 2)$ resp. $V_{3}^{6}(\boldsymbol{C}, 2)$ are not closed in $V_{3}^{6}(\boldsymbol{R})$ resp. $V_{3}^{6}(\boldsymbol{C})$.

Theorem (3.2) whose proof used (5.10) is therefore completely established. 
We now prove a surprising fact for $C$ which has no analogue for $R$ (see (6.3)):

(5.11) The interior of $W_{3}^{6}(\boldsymbol{C}, 3)$ as a set in $V_{3}^{6}(\boldsymbol{C})$ is empty.

We show that if $\widetilde{R}=R_{1}+R_{2}+R_{3}, R_{i} \in G_{3}^{6}(C)$, is irreducible then it is the limit of elements in $V_{3}^{6}(C, 2)$.

$R_{i}$ and $R_{j}(i \neq j)$ have no nonvanishing 2 -vector as a common factor, because $R_{i}+R_{j}$ would then be simple. Thus two cases are to be considered:

(1) $R_{i} \wedge R_{j} \neq 0$ for some $i, j$, say $R_{1} \wedge R_{2} \neq 0$,

(2) $R_{i}$ and $R_{j}$ have for $i \neq j$ a vector $v_{k} \neq 0$ (but no 2 -vector $\neq$ $0)$ as a common factor where $(i, j, k)$ is a permutation of $(1,2,3)$.

In the latter case the $v_{i}$ are either parallel or no two $v_{i}$ are parallel. If they were parallel we could choose $e_{6}$ parallel to the $v_{i}$ so that $\widetilde{R}=\widetilde{S} \wedge e_{6}$ with $\widetilde{S} \in V_{2}^{5}(\boldsymbol{C})$, and $\Sigma R_{i}$ would be reducible since $N(F, 5,2)=2$. If no two $v_{i}$ are parallel then with suitable $u_{i}$

$$
R_{1}=u_{1} \wedge v_{2} \wedge v_{3}, R_{2}=u_{2} \wedge v_{1} \wedge v_{3}, R_{3}=u_{3} \wedge v_{1} \wedge v_{2} .
$$

The vectors $u_{i}, v_{j}$ are independent, for otherwise $\Sigma R_{i}$ would be a 3vector in a space of dimension less than 6 and by $N(F, 5,3)=2$ reducible. The proof of (5.10) shows $\widetilde{R}$ can be approximated by elements of $V_{3}^{6}(C, 2)$.

In case (1) there are vectors $w_{1}, \cdots, w_{6}, v_{1}, v_{2}, v_{3}$ such that $w_{1}, w_{2}$, $w_{3}$ are parallel to $R_{1}, w_{4}, w_{5}, w_{6}$ are parallel to $R_{2}, R_{3}=v_{1} \wedge v_{2} \wedge v_{3}$ and

$$
v_{1}=a_{1} w_{1}+a_{4} w_{4}, v_{2}=a_{2} w_{2}+a_{5} w_{5}, v_{3}=a_{3} w_{3}+a_{6} w_{6} \text {. }
$$

If $\bigwedge_{i=1}^{i} w_{i} \neq 0$ then $\widetilde{R}=\sum_{I \in Y} \alpha^{I} w_{I}$ and $\prod_{i=1}^{6} a_{i} \neq 0$ is equivalent to (5.6), so we have a special case of (5.8) (see [1, p. 83]) and hence $\widetilde{R} \in$ $V_{3}^{6}(C, 2)$ contrary to the hypothesis. If $\Lambda_{i=1}^{6} w_{i}=0$ and/or $\prod_{i=1}^{6} a_{i}=0$ we can choose $w_{i}^{\prime}$ and $a_{i}^{\prime}$ arbitrarily close to $w_{i}$ resp. $a_{i}$ such that $\Lambda_{i=1}^{6} w_{i}^{\prime} \neq 0 \prod_{i=1}^{6} a_{i}^{\prime} \neq 0$ and $\lambda \neq \mu$, so that $\widetilde{R}$ is the limit of elements in $V_{3}^{6}(C, 2)$.

For $k r \leqq n$ let $Z_{r}^{n}(F, k)$ be the set of $\widetilde{R}=\sum_{i=1}^{k} R_{i}$ with $\bigwedge_{i=1}^{k} R_{i} \neq$ 0. Then $Z_{r}^{n}(F, k) \subset W_{r}^{n}(F, k)$ by $(2.10)$.

$$
Z_{r}^{n}(\boldsymbol{R} \operatorname{resp} . \boldsymbol{C}, k) \text { is dense in } V_{r}^{n}(\boldsymbol{R} \operatorname{resp} . \boldsymbol{C}, k) \text {. }
$$

This is nearly obvious: If $\widetilde{R}=\sum_{i=1}^{j} R_{i} \in Z_{r}^{n}(j), j<k$, then $R_{j+1}$, $\cdots, R_{k}$ exist with $\bigwedge_{i=1}^{k} R_{i} \neq 0$ and

$$
\widetilde{R}=\lim \left(\widetilde{R}+\delta \sum_{i=j+1}^{k} R_{i}\right) \text { as } \delta \rightarrow 0 \text {. }
$$

If $\widetilde{R}=\sum_{i=1}^{j} R_{i} \in W_{r}^{n}(j), j \leqq k, \bigwedge_{i=1}^{j} R_{i}=0$ and $R_{i}=\bigwedge_{h=1}^{r} v_{(i-1) r+h}$ then 
$w_{i} \rightarrow v_{i}$ with $\bigwedge_{i=1}^{j r} w_{i} \neq 0$ exist and $\sum_{i=1}^{j} \bigwedge_{h=1}^{r} w_{(i-1) r+h} \rightarrow \widetilde{R}$.

Because

$$
V_{3}^{6}(\boldsymbol{C})=Z_{3}^{6}(\boldsymbol{C}, 2) \cup V_{3}^{6}(\boldsymbol{C}, 2) / Z_{3}^{6}(\boldsymbol{C}, 2) \cup W_{3}^{6}(\boldsymbol{C}, 3),
$$

$(5.11,12)$ show that $Z_{3}^{6}(\boldsymbol{C}, 2)$ is dense in $V_{3}^{6}(\boldsymbol{C})$, so that $V_{3}^{6}(\boldsymbol{C}) / Z_{3}^{6}(\boldsymbol{C}, 2)$ has no interior points, and hence has dimension less than 40 (= $\left.\operatorname{dim} V_{3}^{6}(\boldsymbol{C})\right)$, see [2, p. 46]. In the next section we will see that $Z_{3}^{6}(\boldsymbol{C}, 2)$ is open. Thus

(5.13) The set $Z_{3}^{6}(\boldsymbol{C}, 2)$ is open and dense in $V_{3}^{6}(\boldsymbol{C})$, hence

$$
V_{3}^{6}(C) / Z_{3}^{6}(C, 2)
$$

is closed and $\operatorname{dim} Z_{3}^{6}(\boldsymbol{C}, 2)=40, \operatorname{dim} V_{3}^{6}(\boldsymbol{C}) / Z_{3}^{6}(\boldsymbol{C}, 2)<40$.

Note that $W_{3}^{6}(C, 3) \subset V_{3}^{6}(\boldsymbol{C}) / Z_{3}^{6}(\boldsymbol{C}, 2)$ and that therefore the closure of $W_{3}^{6}(\boldsymbol{C}, 3)$ has dimension less than 40.

6. The sets $Z_{3}^{6}(\boldsymbol{R}$ resp. $\boldsymbol{C}, 2)$ and $W_{3}^{6}(\boldsymbol{R}, 3)$. We now prove that $Z_{3}^{6}(\boldsymbol{R}$ resp. $\boldsymbol{C}, 2)$ is open. Actually our next theorem provides much more information which will allow us to show that $W_{3}^{6}(\boldsymbol{R}, 3)$ has a nonempty interior.

Theorem 6.1. Let $F=\boldsymbol{R}$ or $\boldsymbol{C}$. If $R_{1}, R_{2} \in G_{3}^{6}(F)$ and $R_{1} \wedge R_{2} \neq 0$ then there is a neighborhood $\widetilde{U}\left(\widetilde{R}_{0}\right)$ of $\widetilde{R}_{0}=R_{1}+R_{2}$ in $V_{3}^{6}(F)$ such that for $\widetilde{R} \in \widetilde{U}\left(\widetilde{R}_{0}\right)$ there are simple $R_{1}^{\prime}, R_{2}^{\prime}$ with $\widetilde{R}=R_{1}^{\prime}+R_{2}^{\prime}$. Furthermore, given neighborhoods $U_{i}\left(R_{i}\right)$ of $R_{i}$ in $G_{3}^{6}(F)$ there is a neighborhood $\widetilde{U}^{\prime}\left(\widetilde{R}_{0}\right) \subset \widetilde{U}\left(\widetilde{R}_{0}\right)$ such that $\widetilde{R} \in \widetilde{U}^{\prime}\left(\widetilde{R}_{0}\right)$ implies $R_{i}^{\prime} \in U_{i}\left(R_{i}\right)$ and $R_{1}^{\prime} \wedge R_{2}^{\prime} \neq 0$. Consequently $\widetilde{R} \in Z_{3}^{6}(F, 2)$ and by (4.4) $R_{1}^{\prime}+R_{2}^{\prime}$ is unique.

Necessary for (6.1) to work is that $G_{3}^{6} \times G_{3}^{6}$ and $V_{3}^{6}$ have the same dimension, which is the case because $a$-dim $G_{r}^{n}=r(n-r)+1$, $a-\operatorname{dim} V_{r}^{n}=\left(\begin{array}{l}n \\ r\end{array}\right)$ and $2[3(6-3)+1]=20=\left(\begin{array}{l}6 \\ 3\end{array}\right)$. But this argument is far from sufficient as similar situations for other dimensions show; the structure of $G_{3}^{6}$ enters.

Since $R_{1} \wedge R_{2} \neq 0$ we can choose a base so that $R_{1}=e_{123}, R_{2}=e_{456}$. A neighborhood of $R_{1}$ on $G_{3}^{6}$ consists of the simple $R_{1}^{\prime}=\Sigma \beta^{I} e_{I}=$ $\sum_{1 \leqq i<j<k \leqq 6} \beta^{i j k} e_{i j k}$ with $\beta^{123}$ close to 1 and the remaining $\beta^{I}$ close to 0 , so $\beta^{123} \neq 0$ may be assumed. Similarly for $R_{2}^{\prime}=\Sigma \gamma^{I} e_{I}$ and $\gamma^{456} \neq 0$. The components of $\widetilde{R}_{0}$ are $1,0, \cdots, 0,1$.

The special properties of $G_{3}^{6}$ arise from the Plücker relations which (see [1, p. 69]) with $\lambda=\left(\beta^{123}\right)^{-1}$ are equivalent to 


$$
\begin{aligned}
\beta^{i j k}= \pm \lambda\left|\begin{array}{ll}
\beta^{i \rho j} & \beta^{i \rho k} \\
\beta^{i \sigma j} & \beta^{i \sigma k}
\end{array}\right| 1 \leqq i \leqq 3,4 \leqq j<k \leqq 6,(i, \rho, \sigma)=\pi(1,2,3), \\
\beta^{456}=\lambda^{2}\left|\begin{array}{lll}
\beta^{124} & \beta^{125} & \beta^{126} \\
\beta^{134} & \beta^{135} & \beta^{136} \\
\beta^{234} & \beta^{235} & \beta^{236}
\end{array}\right|=B .
\end{aligned}
$$

Similarly with $\mu=\left(\gamma^{456}\right)^{-1}$

$$
\begin{aligned}
\gamma^{i j k}= \pm \mu\left|\begin{array}{ll}
\gamma^{i \rho k} & \gamma^{j \rho k} \\
\gamma^{i \sigma k} & \gamma^{j \sigma k}
\end{array}\right| 1 \leqq i<j \leqq 3,4 \leqq k \leqq 6,(\rho, \sigma, k)=\pi(4,5,6), \\
\gamma^{123}=\mu^{2}\left|\begin{array}{lll}
\gamma^{145} & \gamma^{245} & \gamma^{345} \\
\gamma^{146} & \gamma^{246} & \gamma^{346} \\
\gamma^{156} & \gamma^{256} & \gamma^{356}
\end{array}\right|=C .
\end{aligned}
$$

If now $\Sigma \alpha^{I} e_{I}=\widetilde{R}=R_{1}^{\prime}+R_{2}^{\prime}=\Sigma \beta^{I} e_{I}+\Sigma \gamma^{I} e_{I}$ then $\alpha^{I}=\beta^{I}+\gamma^{I}$, and substitution gives

$$
\begin{aligned}
& \alpha^{123}=\beta^{123}+C, \\
& \alpha^{i j k}=\beta^{i j k} \pm \mu\left|\begin{array}{ll}
\gamma^{i \rho k} & \gamma^{j \rho k} \\
\gamma^{i \sigma k} & \gamma^{j o k}
\end{array}\right| 1 \leqq i<j \leqq 3,4 \leqq k \leqq 6,(\rho, \sigma, k)=\pi(4,5,6),
\end{aligned}
$$$$
\alpha^{i j k}= \pm \lambda\left|\begin{array}{ll}
\beta^{i \rho j} & \beta^{j \rho k} \\
\beta^{i \sigma j} & \beta^{i \sigma k}
\end{array}\right|+\gamma^{i j k}, 1 \leqq i \leqq 3,4 \leqq j<k \leqq 6,(i, \rho, \sigma)=\pi(1,2,3) \text {, }
$$$$
\alpha^{456}=B+\gamma^{456} \text {. }
$$

Thus the 20 components of $\widetilde{R}$ are expressed in terms of $\beta^{123}$, the nine $\beta^{I}$ with $1 \leqq i \leqq 3,4 \leqq j<k \leqq 6$, the nine $\gamma^{I}$ with $1 \leqq i<j \leqq 3,4 \leqq$ $k \leqq 6$, and $\gamma^{456}$. Evaluation of the functional determinant at $(1,0, \cdots$, $0,1)$ gives the value 1 . Therefore, the implicit function theorem is applicable and yields the assertion. The details of the calculation may be found in the thesis, [1, pp. 93-97].

As a corollary we have

(6.2) The set $Z_{3}^{6}(\boldsymbol{R} \operatorname{resp} . \boldsymbol{C}, 2)$ is open in $V_{3}^{6}(\boldsymbol{R}$ resp. $\boldsymbol{C})$.

But in contrast to (5.11):

(6.3) The interior of $W_{3}^{6}(\boldsymbol{R}, 3)$ is not empty.

For take any $\widetilde{R}_{0}=\sum_{I \in Y} \alpha^{I} e_{I}$ of (5.8) for which the $\alpha^{I}$ are real but $\lambda, \mu$ are not. Then

$$
\widetilde{R}_{0}=R_{\beta}+R_{r} \text { with } \quad R_{\beta} \wedge R_{r} \neq 0 .
$$

By (6.1) for $\widetilde{R} \in \widetilde{U}^{\prime}\left(\widetilde{R}_{0}\right) \subset V_{3}^{6}(C)$ 
$\widetilde{R}=R_{1}^{\prime}+R_{2}^{\prime}$ with $R_{1}^{\prime} \wedge R_{2}^{\prime} \neq 0, R_{1}^{\prime}$ close to $R_{\beta}$ and $R_{2}^{\prime}$ close to $R_{r}$ so that $R_{1}^{\prime}$ and $R_{2}^{\prime}$ cannot be real either. Also $R_{1}^{\prime}+R_{2}^{\prime}$ is unique by (4.4) and this implies as in the proof of (5.9) that

$$
\widetilde{R} \in W_{3}^{6}(\boldsymbol{R}, 3) \text { for } \quad \widetilde{R} \in \widetilde{U}^{\prime}\left(\widetilde{R}_{0}\right) \cap V_{3}^{6}(\boldsymbol{R}),
$$

where we consider $V_{3}^{6}(\boldsymbol{R})$ as a subset of $V_{6}^{3}(\boldsymbol{C})$.

\section{REFERENCES}

1. D. E. Glassco, Irreducible Sums of Simple Multivectors, Dissertation, University of Southern California, 1971.

2. W. Hurewicz and H. Wallmann, Dimension Theory, 1st edition, Princeton, 1941.

3. J. Schouten, Tensor Analysis for Physicists, 2nd edition, Oxford, 1954.

4. B. L. Van der Waerden, Einführung in die Algebraische Geometrie, Berlin, 1938, New York, 1945.

Received July 17, 1972.

UNIVERSity OF SOUthern CALIForNia

AND

California Polytechnic State University at San Luis Obispo 


\section{PACIFIC JOURNAL OF MATHEMATICS}

\section{EDITORS}

RICHARD ARENS (Managing Editor) University of California

Los Angeles, California 90024

R. A. Beaumon'T

University of Washington Seattle, Washington 98105
J. Dugundj1*

Department of Mathematics University of Southern California Los Angeles, California 90007

D. Gilbarg and J. Milgram Stanford University Stanford, California 94305

\section{ASSOCIATE EDITORS}

E. F. BECKENBACH

B. H. NeUMANN

F. WOLF

K. YOSHIDA

\section{SUPPORTING INSTITUTIONS}

UNIVERSITY OF BRITISH COLUMBIA CALIFORNIA INSTITUTE OF TECHNOLOGY UNIVERSITY OF CALIFORNIA MONTANA STATE UNIVERSITY UNIVERSITY OF NEVADA NEW MEXICO STATE UNIVERSITY OREGON STATE UNIVERSITY UNIVERSITY OF OREGON OSAKA UNIVERSITY

\section{UNIVERSITY OF SOUTHERN CALIFORNIA STANFORD UNIVERSITY UNIVERSITY OF TOKYO UNIVERSITY OF UTAH WASHINGTON STATE UNIVERSITY UNIVERSITY OF WASHINGTON AMERICAN MATHEMATICAL SOCIETY NAVAL WEAPONS CENTER}

* C. R. DePrima California Institute of Technology, Pasadena, CA 91109, will replace J. Dugundji until August 1974. 


\section{Pacific Journal of Mathematics}

\section{Vol. 49, No. $1 \quad$ May, 1973}

A. Bigard, Free lattice-ordered modules ...........................

Richard Bolstein and Warren R. Wogen, Subnormal operators in strictly cyclic

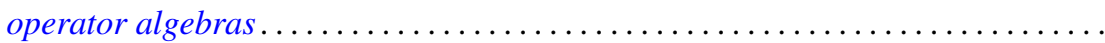

Herbert Busemann and Donald E. Glassco, II, Irreducible sums of simple

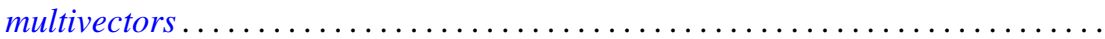

W. Wistar (William) Comfort and Victor Harold Saks, Countably compact groups

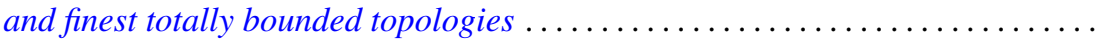

Mary Rodriguez Embry, Maximal invariant subspaces of strictly cyclic operator

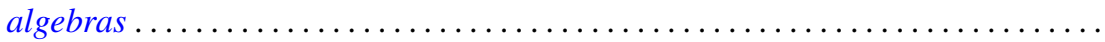

Ralph S. Freese and James Bryant Nation, Congruence lattices of semilattices......

Ervin Fried and George Grätzer, A nonassociative extension of the class of

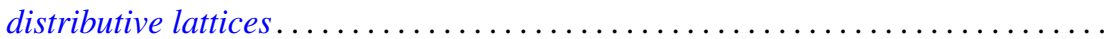

John R. Giles and Donald Otto Koehler, On numerical ranges of elements of locally

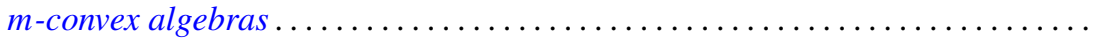

David A. Hill, On dominant and codominant dimension of $\mathrm{QF}-3$ rings ........ John Sollion Hsia and Robert Paul Johnson, Round and Pfister forms over $R(t) \ldots$ I. Martin (Irving) Isaacs, Equally partitioned groups . . . . . . . . . . . . . .

Athanassios G. Kartsatos and Edward Barry Saff, Hyperpolynomial approximation of solutions of nonlinear integro-differential equations.

Shin'ichi Kinoshita, On elementary ideals of $\theta$-curves in the 3-sphere and 2-links in the 4-sphere

Ronald Brian Kirk, Convergence of Baire measures

R. J. Knill, The Seifert and Van Kampen theorem via regular covering spaces ..

Amos A. Kovacs, Homomorphisms of matrix rings into matrix rings ..

Young K. Kwon, HD-minimal but no $H D$-minimal ..........

Makoto Maejima, On the renewal function when some of the mean renewal lifetimes are infinite

Juan José Martínez, Cohomological dimension of discrete modules over profinite groups.

W. K. Nicholson, Semiperfect rings with abelian group of units

Louis Jackson Ratliff, Jr., Three theorems on imbedded prime divisors of principal ideals.

Billy E. Rhoades and Albert Wilansky, Some commutants in $B(c)$ which are almost matrices

John Philip Riley Jr., Cross-sections of decompositions . . .

Keith Duncan Stroyan, A characterization of the Mackey uniformity $m\left(L^{\infty}, L^{1}\right)$ for

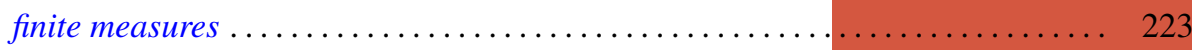

Edward G. Thurber, The Scholz-Brauer problem on addition chains . . . . . . . . . 229

Joze Vrabec, Submanifolds of acyclic 3-manifolds ............

Philip William Walker, Adjoint boundary value problems for compactified singular

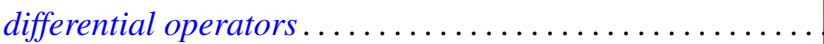

